\title{
Mediterranean dietary pattern and mortality among young women: a cohort study in Sweden
}

\author{
Pagona Lagiou ${ }^{1,2}$, Dimitrios Trichopoulos ${ }^{1,2}$, Sven Sandin $^{3}$, Areti Lagiou ${ }^{1,4}$, Lorelei Mucci ${ }^{2,5}$, \\ Alicja Wolk ${ }^{6}$, Elisabete Weiderpass ${ }^{3,7}$ and Hans-Olov Adami ${ }^{2,3}$ \\ ${ }^{1}$ Department of Hygiene and Epidemiology, University of Athens Medical School, 75 M. Asias Street, Goudi, GR-115 27, Athens, \\ Greece \\ ${ }^{2}$ Department of Epidemiology, Harvard School of Public Health, 677 Huntington Avenue, Boston MA 02115, USA \\ ${ }^{3}$ Department of Medical Epidemiology and Biostatistics, Karolinska Institutet, SE-17177, Stockholm, Sweden \\ ${ }^{4}$ Faculty of Health Professions, Athens Technological Institute (TEI), 274 Thivon Avenue, Athens, Greece \\ ${ }^{5}$ Channing Laboratory, Harvard Medical School, 181 Longwood Avenue, Boston MA 02115, USA \\ ${ }^{6}$ Institute of Environmental Medicine, Karolinska Institutet, Box 210, SE-17177, Stockholm, Sweden \\ ${ }^{7}$ The Cancer Registry of Norway, Montebello, N-0310, Oslo, Norway
}

(Received 14 December 2005 - Revised 23 March 2006 - Accepted 1 April 2006)

\begin{abstract}
Studies of diet and health focus increasingly on dietary patterns. Although the traditional Mediterranean diet is perceived as being healthy, there is little information on its possible benefit to young people. We studied whether closer adherence to the traditional Mediterranean dietary pattern was associated with overall and cancer mortality in a cohort of 42237 young women, aged 30-49 years at enrolment, who were recruited in 1991-2 from the general population in the Uppsala Health Care Region, Sweden, and followed up, almost completely, for about 12 years. Adherence to the Mediterranean diet was assessed by a 10-point score incorporating the characteristics of this diet. Among women less than 40 years old at enrolment - whose causes of death are mainly cancer with probable genetic influences, injuries or suicide - there was no association of the Mediterranean diet score with total or cancer mortality. Among women 40-49 years old at enrolment, a 2-point increase in the score was associated with considerable reductions in overall mortality $(13 \% ; 95 \%$ CI $1 \%, 23 \% ; P \sim 0.05)$ and cancer mortality $(16 \% ; 95 \%$ CI $-1 \%, 29 \%$; $P \sim 0.06)$. Few cardiovascular deaths occurred in this cohort of young women. The findings of the present study in a northern European population of young women indicate that closer adherence to a Mediterranean dietary pattern reduces mortality even among young persons.
\end{abstract}

Mediterranean diet: Sweden: Women: Mortality: Cancer mortality: Diet: Cohort

Keys and his colleagues in the 1960s (Keys, 1980) advanced the hypothesis that the Mediterranean diet might protect against CHD, mainly on account of its low saturated lipid content. In the early 1990s, the emphasis was shifted from the low saturated lipid content of this diet towards its high content of vegetables and legumes and its heavy reliance on unsaturated lipids, largely derived, particularly in the Mediterranean countries, from olive oil (Sacks \& Willett, 1991; Trichopoulou et al. 1995; Willett et al. 1995). Moreover, evidence has accumulated that the Mediterranean diet and its key components, including monounsaturated lipids, may have beneficial effects not only on CHD, but also on some forms of cancer and, eventually, on longevity itself (Wolk et al. 1998, Trichopoulou et al. 2000; Bosetti et al. 2003). The study of the association of the Mediterranean diet, as an integral dietary pattern, with various indicators of health and disease was facilitated by its operationalisation through a Mediterranean diet score (Trichopoulou et al. 1995), which assesses adherence to this dietary pattern and has been used extensively, in different variants, by several authors in many investigations in
Mediterranean and non-Mediterranean countries (Osler \& Schroll, 1997; Kouris-Blazos et al. 1999; Lasheras et al. 2000; Bosetti et al. 2003; Chrysohoou et al. 2004; Trichopoulou et al. 2005b).

There is now considerable evidence that a Mediterranean dietary pattern increases longevity among the elderly (Trichopoulou et al. 2003; Knoops et al. 2004). The effect of the Mediterranean diet on total mortality among younger persons, however, has not been extensively studied, because of obvious limitations in statistical power (few outcomes). In the study by Trichopoulou et al. (2003), no significant association of the Mediterranean diet score with overall mortality was evident among persons who were younger than 50 years at enrolment, possibly because there were fewer than fifty deaths in this group. There is therefore a genuine question over whether the Mediterranean diet is associated with reduced mortality among younger persons, because among this group mortality is dominated by cancer, as well as causes that are unlikely to be related to diet, that is, injuries, suicides and infections. 
We have investigated the association of the Mediterranean dietary pattern with overall and cancer mortality in a large cohort of Swedish women younger than 50 years at enrolment. These women were further distinguished into those who were 40 years or older and those who were less than 40 years at enrolment. In the latter group, very few deaths are of a cardiovascular nature, cancer has frequently strong genetic determinants (Loman et al. 2001; Hoedema et al. 2003; Li and Hemminki 2004), and many deaths are due to accidents and self-inflicted injuries, so that diet is unlikely to play an important role. Among women 40 years or older at enrolment, however, diet in general and the Mediterranean diet in particular could be an important determinant of mortality. Hence, the association of the Mediterranean dietary pattern with overall and cancer mortality was investigated separately for women who were 40 years or older, and younger than 40 years, at enrolment.

\section{Methods}

\section{Recruitment}

Women aged 30-49 years, residing in the Uppsala Health Care Region in Sweden in 1991-2, form the source population for this study. Of this source population, 96000 women were randomly selected from four age strata (30-34, $35-39,40-44$ and $45-49$ years) and were invited to participate in the Swedish component of the Scandinavian Women's Lifestyle and Health Cohort (Veierod et al. 2003). All women received a letter inviting them to participate in the study. The women were asked to fill in a questionnaire and return it in a pre-paid envelope. Of the women invited, over half decided to participate, and 49261 returned the questionnaires and were enrolled in the study. The Swedish Data Inspection Board and the regional Ethical Committee approved the study.

\section{Questionnaire and dietary assessment}

The women filled in a self-administered questionnaire that included information on lifestyle variables (including a detailed smoking history and alcoholic drinking habits), anthropometry (height, weight, BMI), and medical history (previous diagnoses of major diseases). In this questionnaire, women also rated their overall level of physical activity (including activities in the house, occupational and recreational physical activity) on a 5-point scale with examples attached to levels 1, 3 and 5: $1=$ sedentary (mainly sitting); $3=$ moderate physical activity (a few walks a week); $5=$ vigorous physical activity (sports/jogging several times a week). Dietary habits during the 6 months preceding the woman's enrolment in the study were ascertained through a validated food-frequency questionnaire that covered the frequency of consumption and quantity of about eighty food items and beverages (Wolk et al. 1998). Food consumption was translated into nutrient and energy intakes on the basis of the Swedish National Food Administration database (Bergstrom et al. 1991). A total of eleven food groups were constructed (all measured for each woman in $\mathrm{g} / \mathrm{d}$ unless otherwise specified): vegetables, legumes, fruits and nuts, dairy products, cereals, meat and meat products, fish and seafood, potatoes, eggs, sugars and sweets, and non-alcoholic beverages (in $\mathrm{ml} / \mathrm{d}$ ). In addition, the intake of polyunsaturated, monounsaturated and saturated lipids (all in g/d), as well as the ratio between the latter two lipid types, the monounsaturated:saturated ratio, were calculated.

\section{Mediterranean diet score}

To assess adherence to the traditional Mediterranean diet, we used the scale developed by Trichopoulou and colleagues (Trichopoulou et al. 1995, 2003). The scale incorporates information on the consumption of seven food groups - vegetables, legumes, fruits and nuts, cereals, fish and seafood, meat and meat products, and milk and dairy products - as well as on alcohol consumption and the ratio of monounsaturated to saturated lipids in the diet. Using the median consumption as a cut-off, a value of 0 or 1 was assigned to each of the nine indicated components. For components presumed to be beneficial (vegetables, legumes, fruits, cereals, fish), persons whose consumption was below the median were assigned a value of 0 , and persons whose consumption was at or above the median were assigned a value of 1 . For components presumed to be detrimental (meat, dairy products), those whose consumption was below the median were assigned a value of 1 , and those whose consumption was at or above the median were assigned a value of 0 . For alcohol, a value of 1 was assigned to women who consumed between 5 and $25 \mathrm{~g} / \mathrm{d}$. Thus, the total Mediterranean diet score ranged from 0 (minimal adherence to the traditional Mediterranean diet) to 9 (maximal adherence).

\section{Follow-up}

Follow-up of the cohort was achieved through linkages with existing Swedish nationwide health registers. Because each resident in Sweden has a unique national registration number, one can link the data from the cohort with these registers for virtually complete follow-up with respect to death and emigration. From the Register of Total Population, we received information on dates of death for women who died during the follow-up period until 31 December 2003. Additional information on cause of death was derived from the Death Register and was updated till 31 December 2002. Dates of emigration for women who moved out of Sweden were provided by the Register of Total Population. The start of follow-up was defined as the date of return of the questionnaire during 1991-2. Observation time was calculated from the date of entry into the cohort until the occurrence of death, or censoring. For overall mortality, censoring was on account of emigration or the end of the observation period, whereas for cancer mortality, it was also on account of death from any other cause.

\section{Statistical analysis}

Of the original 49261 Swedish women, the following were sequentially excluded: those who had emigrated without reimmigration prior to the start of the study (sixteen women), those who had not filled in the dietary questionnaire (583 women), those with prevalent cancer (excluding non-melanoma skin cancer), CHD or diabetes at enrolment (1418 
women) and those with missing information on any of the covariates studied (4404 women), as well as those with energy intakes outside the first $(1847 \mathrm{~kJ} / \mathrm{d})$ and 99 th $(12474 \mathrm{~kJ} / \mathrm{d})$ centiles (603 women). Thus, a total of 42237 women were available for the analysis.

The participating women, the person-time contributed and the deaths were initially grouped into categories of non-nutritional covariates including age, education, height, BMI, physical activity, smoking and alcohol intake. The associations between the individual covariates and overall mortality were examined with Cox's proportional hazards regression. The associations between these covariates and the Mediterranean diet score were examined by cross-tabulations and Spearman's rank correlation coefficients. The quartile values of food group and macronutrient intake were also determined, and Spearman's rank correlation coefficients relating the Mediterranean diet score to the food group and macronutrient intakes were calculated

Hazard ratios for overall mortality and mortality from cancer were estimated through Cox proportional hazards regression, using the Mediterranean diet score as the principal exposure. Mortality from CVD was not studied because, among these young women, very few deaths were due to these conditions. The models were adjusted for the following variables as reported at enrolment: age (years, continuously), height $(\mathrm{cm}$, continuously), BMI $(<25,25-29.99, \geq 30 \mathrm{~kg} /$ $\mathrm{m}^{2}$, categorically), smoking status (never smokers, former smokers of four or fewer cigarettes/d former smokers of 59 cigarettes, former smokers of 10-14 cigarettes, former smokers of 15-19 cigaretted, former smokers of 20 or more cigarettes, current smokers of four or fewer cigarettes, current smokers of 5-9 cigarettes, current smokers of 10-14 cigarettes, current smokers of 15-19 cigarettes, current smokers of 20 or more cigarettes, categorically), physical activity (from 1:low to 5:high, categorically), education $(0-10,11-$ 13,14 or more years in school, categorically) and energy intake (continuously). They were also adjusted for the nutritional variables that were not part of the Mediterranean diet score, specifically potato intake (continuously), egg intake (continuously), polyunsaturated lipid intake (continuously), sweet and sugar intake (continuously) and non-alcoholic beverage intake (continuously). Fine control for tobacco smoking was necessary because of the powerful influence of smoking on mortality and the possibility that smoking might be associated with some dietary intakes. Moreover, control for foods that are not part of the Mediterranean diet score, which has minimal effect on statistical power, was deemed necessary to assure minimisation of possible conditional confounding.

All analyses were conducted for all women, as well as separately for women less than 40 years old at enrolment and for women 40 years or older at enrollment. This decision was made a priori because, among younger women, malignancies frequently have strong genetic determinants (Loman et al. 2001; Hoedema et al. 2003; Li and Hemminki, 2004) and many deaths are due to accidents and self-inflicted injuries, so that diet is unlikely to play an important role.

Statistical analyses were performed using the statistical software $\mathrm{R}$ version 2.0.1 ( $\mathrm{R}$ Project for Statistical Computing, 2006). The Cox proportional hazards assumption was checked informally by graphs of scaled Schoenfeldt residuals $v$. time (Therneau \& Grambsch, 2001). None of the model covariates showed a deviation from the proportional hazards assumption, and no evidence of co-linearity was detected.

\section{Results}

The 42237 women were followed up for an average of 12.01 years, during which time 572 deaths were recorded. Table 1 shows the distribution of participating women, the contributed person-years and the recorded deaths, by sociodemographic, anthropometric and lifestyle variables. Age and mutually adjusted mortality ratios are also presented. The mortality patterns are in line with expectations by indicating that mortality increases with age and BMI, declines with years of schooling and physical activity, and is higher among smokers and heavy drinkers (although the results on alcohol drinking were based on only nine deaths among heavily drinking women).

In Table 2, the women are cross-classified by the previously indicated non-nutritional variables and three categories of the Mediterranean diet score. The score tends to be higher among older, more educated, physically more active, currently non-smoking and moderately drinking women. Thus, all these variables had the potential to confound the association of the Mediterranean diet score with mortality and were therefore controlled for in the analyses.

Table 3 presents the median values and quartiles of the nutritional variables studied and the association of these variables with the Mediterranean diet score. As expected, vegetables, legumes, fruits, cereals, fish and monounsaturated:saturated lipid ratio were positively associated with the Mediterranean diet score, whereas the intakes of dairy products and saturated lipids were inversely associated with this score. Energy intake was positively associated with the Mediterranean diet score, underlying the need to control for this variable in all analyses. Meat intake (a 'detrimental' component) was essentially unrelated to the score, probably because meat contributed about $30 \%$ of the monounsaturated lipid intake in the diet of the study participants (Wolk et al. 1998) and intake of monounsaturated lipids is considered beneficial in the Mediterranean diet score.

Although deaths were recorded until the end of 2003, causes of death were available only for deaths that occurred up until the end of 2002. Table 4 shows the distribution of deaths by age and cause. Cancer was responsible for over $50 \%$ of all deaths, whereas there were very few deaths from CHD, making it unrealistic to explore their relation with diet. About one third of all deaths and nearly $50 \%$ of deaths among the very young women were due to infections, injuries, suicide or other causes that were unlikely to be related to diet. Thus, only total and cancer mortality could be investigated.

The distribution of the 280 cancer deaths by site were as follows: breast, sixty-one; lung, fifty-four; ovaries, twentynine; pancreas, nineteen; colorectal, nineteen; individually less common cancers, ninety-eight (brain, thirteen; various forms of leukaemia and lymphoma, thirteen; stomach, eleven; biliary tract, eleven; uterus, ten; urinary tract, ten; melanoma, seven; oral cavity, five; connective tissue, five; other, seven; unknown, primary seven). Moreover, because among very young women cancer frequently has a genetic basis and causes unrelated to diet dominate their mortality pattern, we particularly focused on women who were 40 years or older at enrolment. Among these women, there were forty-two 
Table 1. Distribution of 42237 Swedish women by non-nutritional characteristics and alcohol intake at enrolment, and corresponding mortality ratios (with $95 \% \mathrm{Cl})^{*}$

\begin{tabular}{|c|c|c|c|c|c|}
\hline Variables & $n$ & Woman-years & Number of deaths & $\begin{array}{l}\text { Age-adjusted mortality } \\
\text { ratios }(95 \% \mathrm{Cl})\end{array}$ & $\begin{array}{l}\text { Multivariate† mortality } \\
\text { ratios }(95 \% \mathrm{Cl})\end{array}$ \\
\hline \multicolumn{6}{|c|}{ Age at enrolment (years) } \\
\hline $29-34$ & 10251 & 122935 & 60 & 1.00 & 1.00 \\
\hline $35-39$ & 10898 & 130959 & 113 & $1.77(1.29,2.42)$ & $1.78(1.30,2.43)$ \\
\hline $40-44$ & 11119 & 133724 & 173 & $2.65(1.98,3.56)$ & $2.53(1.88,3.40)$ \\
\hline $45-49$ & 9969 & 119706 & 226 & $3.87(2.91,5.14)$ & $3.48(2.59,4.65)$ \\
\hline$P$ for trend & & & & $<0.001$ & $<0.001$ \\
\hline \multicolumn{6}{|l|}{ Education (years) } \\
\hline $0-10$ & 12537 & 150997 & 265 & 1.00 & 1.00 \\
\hline $11-13$ & 16418 & 197547 & 183 & $0.67(0.55,0.82)$ & $0.73(0.61,0.90)$ \\
\hline$>13$ & 13282 & 158781 & 124 & $0.52(0.42,0.65)$ & $0.63(0.50,0.78)$ \\
\hline$P$ for trend & & & & $<0.001$ & $<0.001$ \\
\hline \multicolumn{6}{|l|}{ Height (cm) } \\
\hline$<160$ & 5239 & 62675 & 90 & 1.00 & 1.00 \\
\hline $160-164.9$ & 11920 & 143212 & 159 & $0.78(0.60,1.00)$ & $0.82(0.63,1.06)$ \\
\hline $165-169 \cdot 9$ & 13538 & 162756 & 186 & $0.82(0.64,1.05)$ & $0.88(0.68,1.13)$ \\
\hline$\geq 170$ & 11540 & 138682 & 137 & $0.73(0.56,0.96)$ & $0.81(0.62,1.06)$ \\
\hline$P$ for trend & & & & 0.07 & 0.29 \\
\hline \multicolumn{6}{|l|}{ BMI $\left(\mathrm{kg} / \mathrm{m}^{2}\right)$} \\
\hline$<25$ & 30663 & 368257 & 366 & 1.00 & 1.00 \\
\hline $25-29 \cdot 9$ & 9234 & 111052 & 144 & $1.19(0.98,1.45)$ & $1.08(0.88,1.31)$ \\
\hline$\geq 30$ & 2340 & 28016 & 62 & $2.01(1.53,2.63)$ & $1.66(1.26,2.19)$ \\
\hline$P$ for trend & & & & $<0.001$ & 0.003 \\
\hline \multicolumn{6}{|l|}{ Physical activity } \\
\hline 1 (low) & 1724 & 20507 & 50 & 1.00 & 1.00 \\
\hline 2 & 4496 & 53843 & 81 & $0.63(0.44,0.89)$ & $0.75(0.52,1.06)$ \\
\hline 3 & 25183 & 302909 & 338 & $0.46(0.34,0.62)$ & $0.56(0.41,0.76)$ \\
\hline 4 & 7227 & 86728 & 75 & $0.36(0.25,0.52)$ & $0.51(0.35,0.73)$ \\
\hline 5 (high) & 3607 & 43338 & 28 & $0.29(0.18,0.45)$ & $0.39(0.25,0.63)$ \\
\hline$P$ for trend & & & & $<0.001$ & $<0.001$ \\
\hline \multicolumn{6}{|c|}{ Smoking at enrolment } \\
\hline Never smoker & 17427 & 209536 & 160 & 1.00 & 1.00 \\
\hline Ex-smoker & 12476 & 149948 & 158 & $1.35(1.09,1.69)$ & $1.34(1.08,1.68)$ \\
\hline Current smoker & 12334 & 147840 & 254 & $2.32(1.90,2.82)$ & $2.07(1.69,2.54)$ \\
\hline$P$ for trend & & & & $<0.001$ & $<0.001$ \\
\hline \multicolumn{6}{|l|}{ Alcohol intake $(\mathrm{g} / \mathrm{d})$} \\
\hline$<5$ & 31453 & 378110 & 415 & $1.00(0.83-1.21)$ & $1.01(0.84,1.23)$ \\
\hline $5-25$ & 10595 & 126982 & 148 & 1.00 & 1.00 \\
\hline$>25$ & 189 & 2232 & 9 & $3.29(1.68-6.38)$ & $2.59(1.32,5.09)$ \\
\hline$P$ for trend & & & & 0.45 & 0.41 \\
\hline
\end{tabular}

${ }^{*}$ Calculated by Cox proportional hazards regression.

$\dagger$ Adjusted for the variables in this table.

deaths from breast cancer, forty-five from lung cancer, twentyfive from ovarian cancer, fifteen from colorectal cancer, fifteen from pancreatic cancer and sixty-two from individually less common cancers.

Table 5 summarises the association of the Mediterranean diet with overall mortality and mortality from cancer in the total group of women, controlling for potential confounders. For total as well as for cancer mortality, results are presented for both the ordinal increase in the score (per 2 units) and for score categories (0-3 as reference, 4-5 and 6-9 units). An increasing Mediterranean diet score is associated with reduced overall mortality, and the reduction is largely accounted for by cancer mortality. In the fully adjusted models, however, neither of the two examined trends was statistically significant.

In Table 6, we evaluated separately the association of the score with overall and cancer mortality among very young women and women aged 40-49 years at enrolment. There was no association between the score and either total or cancer mortality among the very young women. Among women 40 years or older at enrolment, however, the Mediterranean diet score was associated with substantial reductions in both overall $(P<0.05)$ and cancer $(P \sim 0.06)$ mortality. The interaction term assessing the difference in total mortality ratio among younger and older women $(1.09 v .0 .87)$ is highly suggestive $(P \sim 0.056)$. The interaction term assessing the difference in cancer mortality ratio among younger (1.07) and older $(0.84)$ women is clearly not statistically significant $(P \sim 0.184)$. The contrast of the ratios, however, is at least as striking for cancer as for total mortality, indicating that small numbers are possibly responsible for the lack of statistical significance.

Figure 1 shows Cox model-derived survival curves among women aged 40-49 years at enrolment by Mediterranean diet score category. It illustrates the substantial association of the Mediterranean diet score with overall mortality among women 40-49 years old at enrolment, largely accounted for by a reduction in mortality from cancer. 
Table 2. Distribution of 42237 Swedish women participants by Mediterranean diet score in conjunction with non-nutritional variables and alcohol intake

\begin{tabular}{|c|c|c|c|c|c|c|}
\hline & \multicolumn{6}{|c|}{ Mediterranean diet score } \\
\hline & \multicolumn{2}{|c|}{$0-3$} & \multicolumn{2}{|c|}{$4-5$} & \multicolumn{2}{|c|}{$6-9$} \\
\hline & $n$ & $\%^{*}$ & $n$ & $\%^{*}$ & $n$ & $\%$ * \\
\hline \multicolumn{7}{|l|}{ Age at enrolment (years) } \\
\hline $29-34$ & 4049 & 39 & 4363 & 43 & 1839 & 18 \\
\hline $35-39$ & 3793 & 35 & 4817 & 44 & 2288 & 21 \\
\hline $40-44$ & 3510 & 32 & 4930 & 44 & 2679 & 24 \\
\hline $45-49$ & 2976 & 30 & 4346 & 43 & 2647 & 27 \\
\hline Spearman $r(P$ value $) \dagger$ & \multicolumn{6}{|c|}{$+0.10(<0.001)$} \\
\hline \multicolumn{7}{|l|}{ Education (years) } \\
\hline $0-10$ & 4873 & 39 & 5269 & 42 & 2395 & 19 \\
\hline $11-13$ & 5805 & 35 & 7174 & 44 & 3439 & 21 \\
\hline$>13$ & 3650 & 27 & 6013 & 45 & 3619 & 27 \\
\hline Spearman $r(P$ value $) \dagger$ & \multicolumn{6}{|c|}{$+0.12(<0.001)$} \\
\hline \multicolumn{7}{|l|}{ Height $(\mathrm{cm})$} \\
\hline$<160$ & 1896 & 36 & 2298 & 44 & 1045 & 20 \\
\hline $160-164 \cdot 9$ & 4130 & 35 & 5137 & 43 & 2653 & 22 \\
\hline $165-169 \cdot 9$ & 4491 & 33 & 5930 & 44 & 3117 & 23 \\
\hline$\geq 170$ & 3811 & 33 & 5091 & 44 & 2638 & 23 \\
\hline Spearman $r$ ( $P$ value $) \dagger$ & \multicolumn{6}{|c|}{$+0.025(<0.001)$} \\
\hline \multicolumn{7}{|l|}{ BMI $\left(\mathrm{kg} / \mathrm{m}^{2}\right)$} \\
\hline$<25$ & 10418 & 34 & 13269 & 43 & 6976 & 23 \\
\hline $25-29 \cdot 9$ & 3053 & 33 & 4127 & 45 & 2054 & 22 \\
\hline$\geq 30$ & 857 & 37 & 1060 & 45 & 423 & 18 \\
\hline Spearman $r(P$ value $) \dagger$ & \multicolumn{6}{|c|}{$+0.008(0.12)$} \\
\hline \multicolumn{7}{|l|}{ Physical activity } \\
\hline 1 (low) & 795 & 46 & 682 & 40 & 247 & 14 \\
\hline 2 & 1700 & 38 & 1956 & 44 & 840 & 19 \\
\hline 3 & 8797 & 35 & 10998 & 44 & 5388 & 21 \\
\hline 4 & 2051 & 28 & 3235 & 45 & 1941 & 27 \\
\hline 5 (high) & 985 & 27 & 1585 & 44 & 1037 & 29 \\
\hline Spearman $r(P$ value $) \dagger$ & \multicolumn{6}{|c|}{$+0.10(<0.001)$} \\
\hline \multicolumn{7}{|l|}{ Smoking at enrolment } \\
\hline Never smoker & 5495 & 32 & 7864 & 45 & 4068 & 23 \\
\hline Ex-smoker & 3906 & 31 & 5432 & 44 & 3138 & 25 \\
\hline Current smoker & 4927 & 40 & 5160 & 42 & 2247 & 18 \\
\hline Spearman $r(P$ value $) \dagger$ & \multicolumn{6}{|c|}{$-0.07(<0.001)$} \\
\hline \multicolumn{7}{|l|}{ Alcohol intake (g/day) } \\
\hline$<5$ & 12187 & 39 & 13669 & 43 & 5597 & 18 \\
\hline $5-25$ & 2063 & 19 & 4709 & 44 & 3823 & 36 \\
\hline$>25$ & 78 & 41 & 78 & 41 & 33 & 17 \\
\hline Spearman $r(P$ value $) \dagger$ & \multicolumn{6}{|c|}{$+0.23(<0.001)$} \\
\hline Total & 14328 & 34 & 18456 & 44 & 9453 & 22 \\
\hline
\end{tabular}

\section{Discussion}

In a cohort study of 42237 Swedish women, who were 30-49 years old at enrolment and were followed up for an average of 12.01 years, the association between adherence to the Mediterranean diet, operationalised through a 10-point score, and overall mortality or mortality from cancer was investigated. Too few of these young women died from CHD or stroke to allow meaningful investigation of a possible association of these conditions with diet. Among the 21149 women who were less than 40 years old at enrolment, no association was found between the Mediterranean diet score and either overall mortality or mortality from cancer. In contrast, among the 21088 women who were 40-49 years old at enrolment, the Mediterranean diet score was inversely associated with both overall mortality and mortality from cancer.
The lack of an association of the Mediterranean dietary pattern, or indeed any aspect of diet, with mortality among very young women is not surprising. Among the 153 deaths for which the cause was recorded, only thirteen were due to CVD, whereas sixty-four deaths were from causes that were unlikely to have nutritional aetiological components (injuries, suicide, infections, etc.). Cancer was responsible for seventy-six deaths, but it is well known that cancer in very young women is frequently under strong genetic influence (Loman et al. 2001; Hoedema et al. 2003; Li and Hemminki, 2004).

The inverse associations of the Mediterranean diet score with both overall mortality and mortality from cancer among women aged 40-49 years at enrolment, who were at their mid-fifties by the end of follow-up, are findings with 
Table 3. Daily dietary intakes of food groups and selected macronutrients, and Spearman correlation coefficients with Mediterranean diet score

(Data from 42237 Swedish women)

\begin{tabular}{|c|c|c|c|c|}
\hline & 25th centile & Median & 75th centile & Spearman's $r^{\star}$ \\
\hline Vegetables (g/d) & $39 \cdot 9$ & 61.9 & $89 \cdot 1$ & +0.54 \\
\hline Legumes $(g / d)$ & $4 \cdot 0$ & $17 \cdot 5$ & $26 \cdot 2$ & +0.45 \\
\hline Fruits and nuts $(\mathrm{g} / \mathrm{d})$ & $81 \cdot 1$ & $136 \cdot 9$ & $209 \cdot 0$ & +0.45 \\
\hline Dairy products (g/d) & $161 \cdot 4$ & $334 \cdot 2$ & $507 \cdot 4$ & -0.29 \\
\hline Cereals $(g / d)$ & $136 \cdot 1$ & $183 \cdot 3$ & $241 \cdot 0$ & +0.27 \\
\hline Meat $(\mathrm{g} / \mathrm{d})$ & $62 \cdot 0$ & 84.4 & $109 \cdot 8$ & +0.02 \\
\hline Fish $(g / d)$ & $14 \cdot 1$ & $22 \cdot 7$ & 31.6 & +0.48 \\
\hline Monounsaturated to saturated lipid intake ratio & 0.68 & 0.75 & 0.83 & +0.37 \\
\hline Potatoes $(\mathrm{g} / \mathrm{d})$ & $47 \cdot 4$ & $71 \cdot 3$ & $102 \cdot 2$ & +0.06 \\
\hline Eggs $(g / d)$ & $6 \cdot 6$ & $14 \cdot 1$ & $14 \cdot 1$ & $+0 \cdot 17$ \\
\hline Sweets $(g / d)$ & $14 \cdot 8$ & $29 \cdot 8$ & $50 \cdot 9$ & +0.09 \\
\hline Non-alcoholic beverages (ml/d) & $499 \cdot 0$ & $695 \cdot 7$ & $905 \cdot 9$ & +0.03 \\
\hline Monounsaturated lipids ( $\mathrm{g} / \mathrm{d})$ & $13 \cdot 7$ & $17 \cdot 6$ & $22 \cdot 1$ & +0.04 \\
\hline Saturated lipids (g/d) & $17 \cdot 6$ & 23.5 & $30 \cdot 5$ & -0.09 \\
\hline Polyunsaturated lipids $(\mathrm{g} / \mathrm{d})$ & $5 \cdot 7$ & $7 \cdot 3$ & $9 \cdot 2$ & +0.19 \\
\hline Energy intake $(\mathrm{kJ} / \mathrm{d})$ & 5213 & 6396 & 7710 & +0.17 \\
\hline
\end{tabular}

${ }^{*}$ All correlations rely on actual values of the respective covariates and are statistically significantly different from zero $(P<0 \cdot 01)$.

Table 4. Deaths by age and cause among Swedish women

\begin{tabular}{|c|c|c|c|c|c|}
\hline Age at enrolment (years) & $\mathrm{CHD} n(\%)$ & Stroke $n(\%)$ & Cancer $n(\%)$ & $\begin{array}{c}\text { Infections, injuries, suicide } \\
\text { and other } n(\%)\end{array}$ & Total $n(\%)$ \\
\hline$<34$ & $0(0.0)$ & $4(8 \cdot 2)$ & $19(38.8)$ & $26(53 \cdot 1)$ & $49(100)$ \\
\hline $35-39$ & $6(5 \cdot 8)$ & $3(2.9)$ & $57(54.8)$ & $38(36.5)$ & $104(100)$ \\
\hline $40-44$ & $7(4.5)$ & $9(5 \cdot 7)$ & $82(52 \cdot 2)$ & $59(37.6)$ & $157(100)$ \\
\hline $45-49$ & $12(6 \cdot 2)$ & $10(5 \cdot 1)$ & $122(62 \cdot 6)$ & $51(26 \cdot 2)$ & $195(100)$ \\
\hline Total & $25(4.9)$ & $26(5 \cdot 1)$ & $280(55.4)$ & $174(34.5)$ & $505(100)$ \\
\hline
\end{tabular}

Cause of death was available for women who died before 31 December 2002. For sixty-seven women who died during 2003, cause of death was not available.

Table 5. Overall and cancer mortality ratios (and $95 \% \mathrm{Cl})^{\star}$ among Swedish women by Mediterranean diet score

\begin{tabular}{|c|c|c|c|}
\hline & \multirow[b]{2}{*}{ Deaths/participants } & \multicolumn{2}{|c|}{ Mortality ratio $(95 \% \mathrm{Cl})$} \\
\hline & & Age-adjusted & Fully adjusted $\dagger$ \\
\hline $\begin{array}{l}\text { Total mortality (trend per } 2 \text { point increase in score) } \\
P \text { for trend }\end{array}$ & $572 / 42237$ & $\begin{array}{l}0.83(0.75,0.92) \\
0.001\end{array}$ & $\begin{array}{l}0.93(0.83,1.03) \\
0.180 \ddagger\end{array}$ \\
\hline \multicolumn{4}{|l|}{ Total mortality (per score category) } \\
\hline Score $0-3$ & $217 / 14328$ & 1 (reference) & 1 (reference) \\
\hline Score $4-5$ & $245 / 18456$ & $0.82(0.69,0.99)$ & $0.93(0.78,1.13)$ \\
\hline Score $6-9$ & $110 / 9453$ & $0.68(0.54,0.86)$ & $0.85(0.67,1.08)$ \\
\hline $\begin{array}{l}\text { Cancer mortality (trend per } 2 \text { point increase in score) } \\
P \text { for trend }\end{array}$ & $280 / 42237$ & $\begin{array}{l}0.82(0.71,0.95) \\
0.015\end{array}$ & $\begin{array}{l}0.89(0.77,1.04) \\
0.200 \ddagger\end{array}$ \\
\hline \multicolumn{4}{|l|}{ Cancer mortality (per score category)§ } \\
\hline Score $0-3$ & $106 / 14328$ & 1 (reference) & 1 (reference) \\
\hline Score 4-5 & $120 / 18456$ & $0.82(0.63,1.07)$ & $0.91(0.69,1.18)$ \\
\hline Score 6-9 & $54 / 9453$ & $0.67(0.48,0.93)$ & $0.80(0.57,1.13)$ \\
\hline
\end{tabular}

* Calculated through Cox proportional hazards regression.

†Adjusted for: age at enrolment (years, continuously), height ( $\mathrm{cm}$, continuously), BMI ( $<25,25-29.99$ and $\geq 30 \mathrm{~kg} / \mathrm{m}^{2}$, categorically), smoking status (never smokers, former smokers of 4 or fewer cigarettes, former smokers of 5-9 cigarettes, former smokers of 10-14 cigarettes, former smokers of 15-19 cigarettes, former smokers of 20 or more cigarettes, current smokers of 4 or fewer cigarettes, current smokers of 5-9 cigarettes, current smokers of 10-14 cigarettes, current smokers of 15-19 cigarettes, current smokers of 20 or more cigarettes, categorically), physical activity (from 1:low to 5:high, categorically), education $(0-10,11-13$ and 14 or more years in school, categorically), energy intake (continuously), potato intake (continuously), egg intake (continuously), polyunsaturated lipid intake (continuously), sweet intake (continuously) and non-alcoholic beverage intake (continuously).

¥ When potato, egg polyunsaturated lipid, sweet and non-alcoholic beverage intake were not adjusted for, the $P$ for trend values for overall and cancer mortality were 0.22 and 0.19 , respectively.

$\S$ Deaths specified to be due to cancer were available only until December 2002. 
Table 6. Total and cancer mortality ratio* among Swedish women associated with a 2-point increase in the Mediterranean diet score by age at enrolment

\begin{tabular}{lccc}
\hline & & \multicolumn{2}{c}{ Mortality ratio $(95 \% \mathrm{Cl})$} \\
\cline { 3 - 4 } & Deaths/participants & Age-adjusted & Fully adjusted $\dagger$ \\
\hline Death from any cause & & & \\
Women $<40$ years & $173 / 21149$ & $0.94(0.78,1.13)$ & $1.09(0.90,1.32)$ \\
Women $\geq 40$ years & $399 / 21088$ & $0.79(0.70,0.89)$ & $0.87(0.76,0.98)$ \\
$P$ for interaction & & & 0.056 \\
Death from cancerł & & $0.92(0.70,1.22)$ & $1.07(0.79,1.43)$ \\
Women $<40$ years & $76 / 21149$ & $0.78(0.66,0.93)$ & $0.84(0.71,1.01)$ \\
Women $\geq 40$ years & $204 / 21088$ & & 0.184 \\
$P$ for interaction & & &
\end{tabular}

${ }^{*}$ Calculated by Cox proportional hazards regression.

$\dagger$ Adjusted as indicated in the second footnote of Table 5. Due to lack of events for the analysis of cancer mortality, the smoking categories $<4$ and 5-9 cigarettes/d were collapsed to a combined category for both former and current smokers.

$\ddagger$ Deaths specified to be due to cancer were available only until December 2002.

potentially important implications. The overall mortality ratio of 0.87 for these women in our study is close to the mortality ratio of 0.89 reported in an earlier Greek study (Trichopoulou et al. 2003) among men and women less than 55 years old at enrolment. It thus appears that the Mediterranean diet has beneficial effects on diseases with nutritional aetiological components, even among individuals in their forties.

In our study, the individual components of the Mediterranean diet score were generally weakly and non-significantly associated with overall mortality and mortality from cancer, even among women older than 40 years at enrolment. This was the case without mutual adjustment among nutritional factors, which was deemed inappropriate because of the underlying complex and co-linear associations between these factors - an important reason for using a score instead. A similar pattern of weak or no associations of mortality with the individual components of the Mediterranean diet score

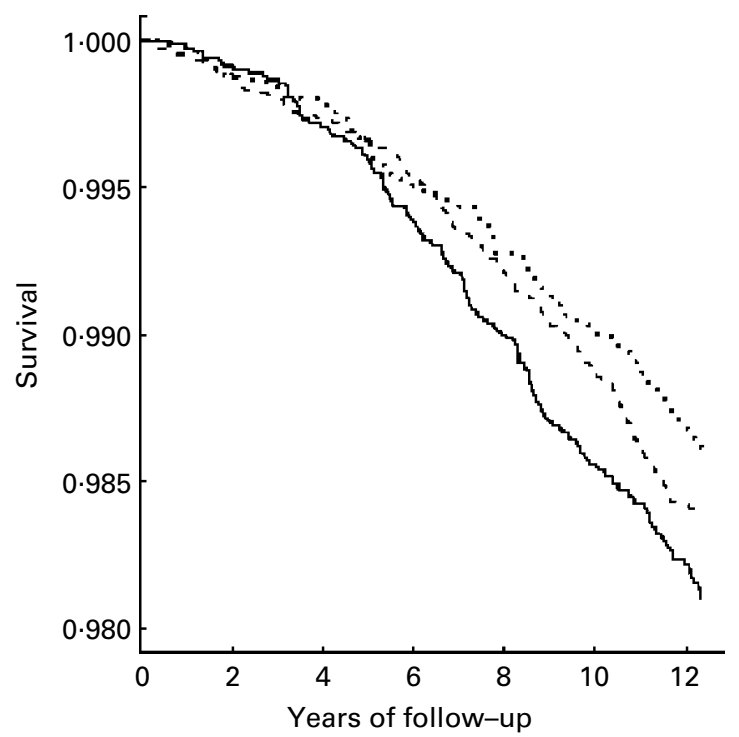

Fig. 1. Cox model-derived survival curves among Swedish women 40-49 years old at enrolment by Mediterranean diet (MD) score categories. __ MD score $0-3 ; \quad$ MD score $4-5 ; . . .$. MD score $6-9$. was also observed in an earlier Greek study (Trichopoulou et al. 2003) in which the score was a significant and important predictor of mortality. With respect to mortality from cancer, recent cohort studies have indicated that two crucial components of the Mediterranean diet - a high intake of vegetables and a high intake of fruits - are weakly associated with a reduced incidence of cancer (Hung et al. 2004) and that a third crucial component of this diet, a low intake of meat, is only weakly associated with reduced colorectal cancer risk (Chan et al. 2005).

Several explanations may apply (Jacques \& Tucker, 2001; Trichopoulou et al. 2003) to the apparent contradiction between the strong associations of an overall dietary pattern and the weak associations of its individual constituents with overall and cancer mortality. First, individual components may have small effects that become apparent and statistically documentable only when the components are integrated into a unidimensional score. Second, there may be biological interactions between one or more components of the score that can only be detected in very large studies, but would still be discernible through a simple unidimensional score. Third, when individual components are evaluated, effects are examined against the background of average risk generated by the other nutritional components, whereas a dietary score accommodates the extremes of cumulative exposure in the absence of other major nutritional effects. In this study, as in earlier ones (Trichopoulou et al. 2005b), the Mediterranean diet score was found to be weakly positively associated with energy intake because more of the score components take a value of 1 with a higher intake of the corresponding food group. This necessitates adjustment for energy intake, but it has trivial consequences on long term BMI-associated conditions (Trichopoulou et al. 2005a).

Although the Mediterranean diet score was developed to assess adherence to the traditional Mediterranean diet (Trichopoulou et al. 1995), it should be recognised that the score incorporates current views about the essence of a healthy diet (Nube et al. 1987; Farchi et al. 1995; Michels \& Wolk, 2002). Indeed, the traditional Mediterranean dietary pattern does not contradict the dietary recommendations of major bodies, except by allowing a higher consumption of lipids, provided they are unsaturated and preferably in the form of 
olive oil (in Greece, for example, the average consumption of olive oil being around $50 \mathrm{~g} / \mathrm{d}$ (Psaltopoulou et al. 2004)). In this context, diets as different as those of Swedes and Greeks can be similarly operationalised, as has been done in this and other investigations (Trichopoulou et al. 2005b).

Strengths of our investigation are its large size, long followup time and minimal losses to follow-up assured by the Register linkage process. Comparability with non-responders is always difficult to assess, but women with missing values were generally similar to those included in the analysis, and, in cohort studies, selection of the study base does not compromise the validity of the results.

Limitations of the study are the unavoidable misclassification of dietary exposures, compounded by the customised classification of the dietary pattern studied and possible changes in dietary habits during the long follow-up period. Nevertheless, these misclassification errors can only attenuate any true association. Because the Mediterranean diet score, as originally introduced and subsequently used (Trichopoulou et al. 1995, 2003, 2005b; Osler \& Schroll, 1997; Kouris-Blazos et al. 1999; Lasheras et al. 2000), did not grade in detail cereals and dairy products, cereals were not distinguished into whole-grain $v$. refined, nor were dairy products separated into high fat $v$. low fat. We have also not distinguished between types of alcoholic beverage because there was not a sufficient number of outcomes to allow the evaluation of different drinking patterns.

Confounding by most known powerful predictors of mortality has been controlled for, but minor or unidentifiable confounders may still operate, and some degree of residual confounding because of misclassification of the recorded confounders cannot be excluded. The residual confounding issue is, however, not specific to this study but haunts all observational investigations.

Using tertiles, quintiles or standardised quantities, instead of the median we employed, might have increased the discriminatory power of the Mediterranean diet score, but it would compromise the simplicity of the message, which is easily conveyed through the use of a median, under the implicit assumption of a monotonic relation. A more detailed quantitative score would have been necessary if one were attempting to explain the fraction of an outcome that could be attributed to various degrees of adherence to the Mediterranean diet, thus focusing, for example, on the components of variance of the outcome linked to a particular exposure. At this stage of research, however, emphasis is on assessment of the usefulness of the Mediterranean diet score in predicting mortality in populations outside the Mediterranean region. Moreover, a main objective of this study was to evaluate whether a dietary pattern approximating the Mediterranean one would be beneficial to young people, as it has been shown to be - through the use of similar instruments in different populations - among older people (Trichopoulou et al. 1995, 2003, 2005b; Osler \& Schroll, 1997; Kouris-Blazos et al. 1999; Lasheras et al. 2000). Finally, the simple score we have used is applicable to most types of dietary questionnaire, including rudimentary ones, thus facilitating replication of the findings in more studies and population groups. We do not argue that a particular pattern in the Swedish diet (defined through the medians in the corresponding components) is Mediterranean. Our working hypothesis has been that the diet of Swedish women would be more conducive to longer survival if it tended to approximate towards the Mediterranean dietary pattern.
In conclusion, we have found evidence that, among women 40-49 years old at enrolment who were followed up for approximately 12 years, closer adherence to a Mediterranean dietary pattern was associated with reduced total mortality and probably mortality from cancer.

\section{Acknowledgements}

The study was supported by grants from the Swedish Cancer Society and the Swedish Research Council.

\section{References}

Bergstrom L, Kylberg E, Hagman U, Eriksson HB \& Bruce A (1991) The food composition database KOST: the National Food Administration's information system for nutritive values of food. Var Foda 43, 439-447.

Bosetti C, Gallus S, Trichopoulou A, Talamini R, Franceschi S, Negri E \& La Vecchia C (2003) Influence of the Mediterranean diet on the risk of cancers of the upper aerodigestive tract. Cancer Epidemiol Biomarkers Prev 12, 1091-1094.

Chan AT, Tranah GJ, Giovannucci EL, Willett WC, Hunter DJ \& Fuchs CS (2005) Prospective study of N-acetyltransferase-2 genotypes, meat intake, smoking and risk of colorectal cancer. Int $J$ Cancer 115, 648-652.

Chrysohoou C, Panagiotakos DB, Pitsavos C, Das UN \& Stefanadis C (2004) Adherence to the Mediterranean diet attenuates inflammation and coagulation process in healthy adults: the ATTICA Study. J Am Coll Cardiol 44, 152-158.

Farchi G, Fidanza F, Grossi P, Lancia A, Mariotti S \& Menotti A (1995) Relationship between eating patterns meeting recommendations and subsequent mortality in 20 years. Eur J Clin Nutr 49, 408-419.

Hoedema R, Monroe T, Bos C, Palmer S, Kim D, Marvin M \& Luchtefeld M (2003) Genetic testing for hereditary nonpolyposis colorectal cancer. Am Surg 69, 387-391.

Hung HC, Joshipura KJ, Jiang R, Hu FB, Hunter D, Smith-Warner SA, Colditz GA, Rosner B, Spiegelman D \& Willett WC (2004) Fruit and vegetable intake and risk of major chronic disease. $J$ Natl Cancer Inst 96, 1577-1584.

Jacques PF \& Tucker KL (2001) Are dietary patterns useful for understanding the role of diet in chronic disease? Am J Clin Nutr 73, $1-2$.

Keys AB (1980) Seven Countries: A Multivariate Analysis of Death and Coronary Heart Disease. Cambridge, MA: Harvard University Press.

Knoops KT, de Groot LC, Kromhout D, Perrin AE, Moreiras-Varela O, Menotti A \& van Staveren WA (2004) Mediterranean diet, lifestyle factors, and 10-year mortality in elderly European men and women: the HALE project. JAMA 92, 433-439.

Kouris-Blazos A, Gnardellis C, Wahlqvist ML, Trichopoulos D, Lukito W \& Trichopoulou A (1999) Are the advantages of the Mediterranean diet transferable to other populations? A cohort study in Melbourne, Australia. Br J Nutr 82, 57-61.

Lasheras C, Fernandez S \& Patterson AM (2000) Mediterranean diet and age with respect to overall survival in institutionalized, nonsmoking elderly people. Am J Clin Nutr 71, 987-992.

Li X \& Hemminki K (2004) Inherited predisposition to early onset lung cancer according to histological type. Int J Cancer 112, 451-457.

Loman N, Johannsson O, Kristoffersson U, Olsson H \& Borg A (2001) Family history of breast and ovarian cancers and BRCA1 and BRCA2 mutations in a population-based series of early-onset breast cancer. J Natl Cancer Inst 93, 1215-1223.

Michels KB \& Wolk A (2002) A prospective study of variety of healthy foods and mortality in women. Int J Epidemiol 31, 847-854. 
Nube M, Kok FJ, Vandenbroucke JP, van der Heide-Wessel C \& van der Heide RM (1987) Scoring of prudent dietary habits and its relation to 25-year survival. J Am Diet Assoc 87, 171-175.

Osler M \& Schroll M (1997) Diet and mortality in a cohort of elderly people in a north European community. Int J Epidemiol 26, 155-159.

Psaltopoulou T, Naska A, Orfanos P, Trichopoulos D, Mountokalakis T \& Trichopoulou A (2004) Olive oil, the Mediterranean diet, and arterial blood pressure: the Greek European Prospective Investigation into Cancer and Nutrition (EPIC) study. Am J Clin Nutr 80, 1012-1018. Erratum Am J Clin Nutr 81, 1181.

R Project for Statistical Computing (2006) www.r-project.org

Sacks FM \& Willett WW (1991) More on chewing the fat. The good fat and the good cholesterol. N Engl J Med 325, 1740-1742.

Therneau TM \& Grambsch PM (2001) Modeling Survival Data: Extending the Cox Model, 2nd edn. Berlin: Springer.

Trichopoulou A, Costacou T, Bamia C \& Trichopoulos D (2003) Adherence to a Mediterranean diet and survival in a Greek population. N Engl J Med 348, 2599-2608.

Trichopoulou A, Kouris-Blazos A, Wahlqvist ML, Gnardellis C, Lagiou P, Polychronopoulos E, Vassilakou T, Lipworth L \& Trichopoulos D (1995) Diet and overall survival in the elderly. BMJ 311, 1457-1460.
Trichopoulou A, Lagiou P, Kuper H \& Trichopoulos D (2000) Cancer and Mediterranean dietary traditions. Cancer Epidemiol Biomarkers Prev 9, 869-873.

Trichopoulou A, Naska A, Orfanos P \& Trichopoulos D (2005a) Mediterranean diet in relation to body mass index and waist-tohip ratio: the Greek European Prospective Investigation into Cancer and nutrition study. Am J Clin Nutr 82, 935-940.

Trichopoulou A, Orfanos P, Norat T, et al. (2005b) Modified-Mediterranean diet and survival: EPIC-elderly prospective cohort study. BMJ 330, 991-997.

Veierod MB, Weiderpass E, Thorn M, Hansson J, Lund E, Armstrong B \& Adami HO (2003) A prospective study of pigmentation, sun exposure, and risk of cutaneous malignant melanoma in women. J Natl Cancer Inst 95, 1530-1538.

Willett WC, Sacks F, Trichopoulou A, Drescher G, Ferro-Luzzi A, Helsing E \& Trichopoulos D (1995) Mediterranean diet pyramid: a cultural model for healthy eating. Am J Clin Nutr 61, Suppl. 6, S1402-S1406.

Wolk A, Bergstrom R, Hunter D, Willett W, Ljung H, Holmberg L, Bergkvist L, Bruce A \& Adami H-O (1998) A prospective study of association of monounsaturated fat and other types of fat with risk of breast cancer. Arch Int Med 158, 41-45. 